\title{
The COVID-19 Pandemic and Mental Health: A Systemic Review
}

\author{
Article by Irin Hossain ${ }^{1}$, Ashekur Rahman Mullick ${ }^{2}$, Ayesha Haidar ${ }^{3}$, M M Aktaruzzaman ${ }^{4}$ \\ ${ }^{1}$ National Institute of Preventive and Social Medicine (NIPSOM), Dhaka, Bangladesh \\ ${ }^{2}$ Delta Medical College and Hospital, Dhaka, Bangladesh \\ ${ }^{3}$ Samorita Hospital Ltd, Dhaka, Bangladesh \\ ${ }^{4}$ DGHS, Mohakhali, Dhaka, Bangladesh \\ E-mail: irin.hossain@gmail.com ${ }^{1}$, ashekmullick@gmail.com ${ }^{2}$
}

\begin{abstract}
This review study aims at discussing about the mental health condition during COVID-19 occurrence among health-care professionals, patients and mass population. The COVID-19 pandemic has been rapidly spread in China, USA, Italy, France, Spain and other Asian and European counterparts. This COVID-19 pandemic has aroused increasing attention nationwide. Patients, health-care professionals, and the mass population are under unmeasurable mental pressure which may lead to different types of mental health problems, such as anxiety, fear, depression, and insomnia. According to the World Health Organization (WHO), mental health includes "subjective well-being, perceived self-efficacy, autonomy, competence, inter-generational dependence, and self-actualization of one's intellectual and emotional potential, among others". The WHO further states that the well-being of an individual is encompassed in the realization of their abilities, coping with normal stresses of life, productive work, and contribution to their community. It was a systemic review study regarding mental health problems due to COVID19. We gather total 29 articles related to COVID-19 and mental health using different search portal like PubMed, Google Scholar, Nature, Lancet. After proper literature review only 8 literatures which were related to this study were taken for this systemic review purpose. Public health and mental health specialist have reached a decision about the severe mental illness during the COVID-19 outbreak among health-care professionals, patients and mass population. But, the rapid spread of the COVID19 has emerged a serious challenge to the mental health service in COVID-19 affected countries.
\end{abstract}

Keywords: COVID-19, Pandemic, Mental Health.

\section{Introduction}

The COVID-19 pandemic has been rapidly spread in China, USA, Italy, France, Spain and other Asian and European counterparts. This COVID-19 pandemic has aroused increasing attention nationwide. Patients, health-care professionals, and the mass population are under unmeasurable mental pressure which may lead to different types of mental health problems, such as anxiety, fear, depression, and insomnia. The COVID-19 outbreak has been rapidly spread in last week of January 2020. The novel coronavirus disease or simply COVID-19 is formerly known as the '2019 Novel Coronavirus (2019-nCoV) Pneumonia', which was originated from a wet market in Wuhan, Hubei province, China in early December 2019. ${ }^{1}$

The corona virus belongs to large family of enveloped RNA virus and causes an infectious disease named coronavirus disease or COVID-19. The COVID-19 virus spreads primarily through droplets of saliva or discharge from the nose when an infected person coughs or sneezes. It may cause various symptoms like high rise of temperature, difficulty in respiration including lung infection and pneumonia like symptoms. On 29 December, 2019 The World Health Organizations (WHO) addressed lower respiratory tract affecting coronavirus as 2019 novel coronavirus which was taken place in Wuhan, China. Later, it named as severe acute respiratory syndrome coronavirus- 2 or simply SARS-Cov- $2 .^{2-3}$

The containment measures during pandemic are:

- Quarantine

- Social distancing and

- Self-isolation

These can have a detrimental impact on mental health, like loneliness and reduced social 
interactions are well-known risk factors for several mental disorders, including schizophrenia and major depression. Public health and mental health specialist have reached a decision about the severe mental illness during the COVID-19 outbreak among health-care professionals, patients and mass population. But, the rapid spread of the COVID-19 has emerged a serious challenge to the mental health service in COVID-19 affected countries.

\section{Materials and Methods}

It was a systemic review study regarding mental health problems due to COVID-19. We gather total 29 articles related to COVID-19 and mental health using different search portal like PubMed, Google Scholar, Nature, Lancet. After proper literature review only 8 literatures which were related to this study were taken for this systemic review purpose.

\section{Discussion}

The mental health and psychosocial consequences of the COVID-19 pandemic may be particularly serious for at least four groups of people:

a) Those who have been directly or indirectly in contact with the virus.

b) Those who are already vulnerable to biological or psychosocial stressors (including people affected by mental health problems).

c) Health professionals (because of higher level of exposure).

d) Even in people who are following the news through numerous media channels.

\section{Mental health issues during the COVID-19 outbreak}

Suspected patients as well as confirmed patients of COVID-19 suffer from various types of psychological pressure and other health related problems. Health-care provider and health professionals are also suffering both physiological and psychological pressure when they take care the infected patients. Not only that, most of the time they come to close contacts with patient's family member or relatives and even facing the public enquiry.

COVID-19 patients both suspected and confirmed cases face many types of mental oriented experience, like

- Fear of severe disease consequences and the contagion.
- Loneliness

- Denial

- Anxiety due to uncertainty about their health status

- Depression

- Insomnia

- Obsessive-compulsive symptoms

- Despair, and

- Suicide.

The poor knowledge regarding COVID-19 and the overwhelming news causes anxiety and fear in the public. Health professionals as well as health-care providers treating COVID-19 patients suffer from,

- Physical exhaustion

- Fear

- Emotion disturbance, and

- Sleep problems.

In a study among 1,563 health professionals showed depressive symptoms, anxiety, and sleep disturbance (Figure 1) ${ }^{1}$.

Mental health disorder Patients in the COVID-19 pandemic

In China, 173 million people are living with mental health disorders. During the time of epidemic, people who have mental health disorders chances to involve more with infection due to,

- Mental health disorders can increase the risk of infections, including pneumonia.

- There is a mark discrimination associated with mental ill health in health care settings, which act as a barrier in accessing timely health service for the mental health disorder patients who are suffering from severe acute respiratory distress due to COVID-19.

- COVID-19 also creates fear, anxiety and depression which stimulates the already worsen mental health condition of mental disorder patients than the normal mass population. ${ }^{4}$

\section{Mental health and psychosocial responses to COVID-19}

It is very natural for any individuals to feel stressed and worried during any pandemic condition. Direct and indirect responses of people affected might include:

- Fear of falling ill and dying

- Avoiding approaching health facilities due to fear of becoming infected while in care 
- Fear of losing livelihoods, not being able to work during isolation, and of being dismissed from work

- Fear of being socially excluded/placed in quarantine because of being associated with the disease (e.g. racism against persons who are from, or perceived to be from, affected areas)

- Feeling powerless in protecting loved ones and fear of losing loved ones because of the virus

- Fear of being separated from loved ones and caregivers due to quarantine regime

- Refusal to care for unaccompanied or separated minors, people with disabilities or the elderly due to fear of infection, because parents or caregivers have been taken into quarantine

- Feelings of helplessness, boredom, loneliness and depression due to being isolated

- Fear of reliving the experience of a previous epidemic

- Health-care providers like nurses, doctors, ambulance drivers, case identifiers, and others are also experience additional stressors during the COVID-19 outbreak, like:

- Stigmatization towards those working with COVID-19 patients and their remains

- Higher demands in the work setting, including long work hours, increased patient numbers and keeping up-to-date with best practices as information about COVID-19 develops

- Reduced capacity to use social support due to intense work schedules and stigma within the community towards frontline workers

- Insufficient personal or capacity to implement basic self-care, especially among people living with a disability

- Insufficient information about the long-term exposure to individuals infected by COVID19

- Fear that health-care providers will pass COVID-19 onto their friends and family as a result of their work.5

\section{Strategy of dealing with mental disorder patients during pandemic}

1. Believing only in reliable source of news like the World Health Organization, the Centers for Disease Control and Prevention, local or regional Public Health Departments.

2. Regular monitor the mental disorder groups as they are not able to visit their health-care providers in regular basis during pandemic time.

3. Take necessary steps to fulfil their basic needs.

4. Teach them to use the social media during the time of pandemic which may reduce the loneliness condition.

5. Advise them to do actively work like walking, exercising, cleaning and organizing household things. ${ }^{6}$

\section{Interventions for healthcare workers with COVID-19 for reducing mental disorders}

1. Treating infection with COVID-19 as a workrelated injury.

2. Managing rotating shift of duty.

3. Teaching the measures for declining infections.

4. Comfortable accommodations for staff who must remain in hospitals

5. Provide adequate amount of PPE

6. Ensure adequate sleep.

7. Access to mental health providers and consultation when required.

8. Learning relaxation techniques and physical exercise

9. Ensure social support. ${ }^{7}$

\section{Strategy of dealing with mental health of children}

1. Help children to cope up with fear by doing extra curriculum activities like drawing.

2. Try to keep children with their parents.

3. Try to maintain familiar routine. ${ }^{8}$

\section{Strategy of dealing with mental health of people in isolation}

1. Maintaining social network through telephone, e-mail, social media and video conference.

2. Try to maintain familiar routine

3. Avoid to ignore the rumors and believing only in reliable source of news. ${ }^{8}$

\section{Conclusion}

The COVID-19 pandemic has caused different types of psychological problems in different group of populations. The COVID-19 pandemic has become an extra socio-economic burden on existing mental health service in world. Finally, there is a need for particular focus on health-care providers. Prevention measures should be taken for reducing secondary traumatic stress reactions, 
including increased awareness of symptoms, help. This will help for managing further need of taking breaks from work, engaging in self-care, mental health disorders during various conditions taking breaks from media coverage and asking for in world.

\section{Figures}

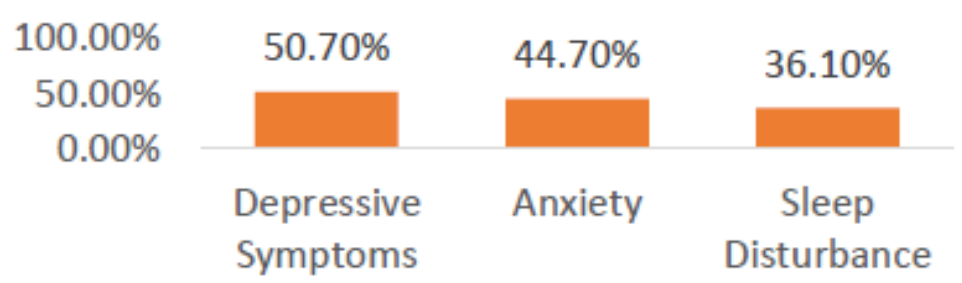

Figure 1. Mental Health Problems

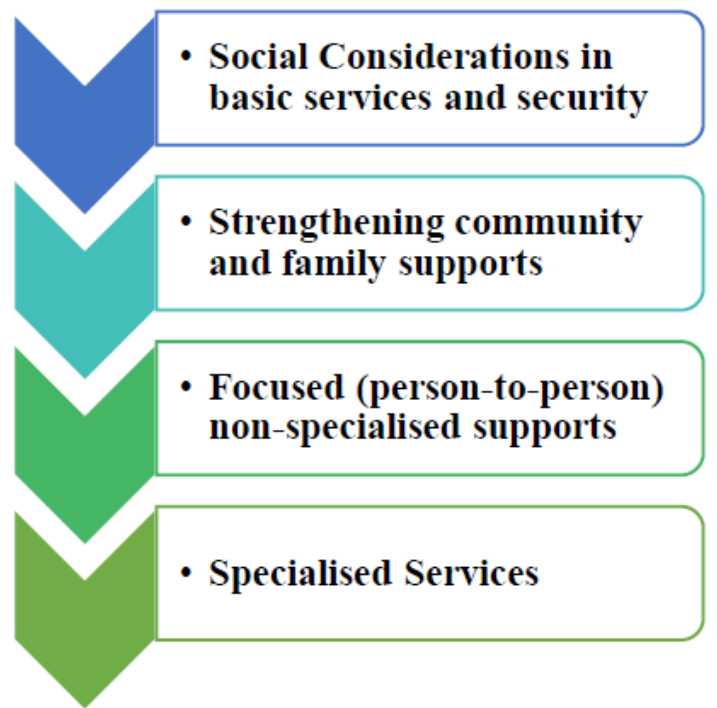

Figure 2. Intervention flowchart for mental health and psychosocial support5

\section{References}

[1]. Li W, Yang Y, Liu Z-H, Zhao Y-J, Zhang Q, Zhang L, et al. Progression of Mental Health Services during the COVID-19 Outbreak in China. Int J Biol Sci [Internet]. 2020;2020(10):1732-8. Available from:

http://www.ijbs.com//creativecommons.org/licenses/ by/4.0/

[2]. World Health Organization. 2020. Coronavirus. Accessed on 27 March, 2020. Available at: https://www.who.int/healthtopics/coronavirustab=tab -1 .

[3]. Chen N, Zhou M, Dong X, Qu J, Gong F, Han Y, et al. Epidemiological and clinical characteristics of 99 cases of 2019 novel coronavirus pneumonia in Wuhan, China: a descriptive study. The Lancet [Internet]. 2020;395(10223):507-13.

[4]. Available from http://dx.doi.org/10.1016/S01406736(20)30211-7

[5]. Yao H, Chen J-H, Xu Y-F. Patients with mental health disorders in the COVID-19 epidemic. The lancet Psychiatry [Internet]. 2020;7(4):e21. Available from:

http://www.ncbi.nlm.nih.gov/pubmed/32199510 [6]. Inter-Agency Standing Committee Reference Group for Mental Health and Psychosocial Support in Emergency Settings. Briefing note on addressing mental health and psychosocial aspects of COVID-19 Outbreak. 2020;(Feb):1-20. Available from: https://app.mhpss.net/?get=354/mhpss-covid19briefing-note-final_26-february-2020.pdf

[7]. SAVE. Preventing Suicide during and After The COVID-19 Pandemic. 2020;

[8]. Mcguire T, Rowe J, Cole K, Herr C, Team HS. Psychosocial Correlates of Outbreak Response Activities: A Supplemental Literature Report. 2020;1-10.ergency PH, Concern I, Health M, Use S. Emergency PH, Concern I, Health M, Use S. Mental Health and Psychosocial Considerations During COVID-19 Outbreak. 2020;(January):1-6. 